\title{
PD-1 and PD-L1 expression in 132 recurrent nasopharyngeal carcinoma: the correlation with anemia and outcomes
}

\author{
Yajuan Zhou ${ }^{1,2, *}$, Jingjing Miao ${ }^{3, *}$, Haijun Wu ${ }^{3}$, Hao Tang ${ }^{4}$, Jing Kuang ${ }^{4}$, Xiaoyi \\ Zhou $^{2}$, Yi Peng ${ }^{2}$, Desheng $\mathrm{Hu}^{2}$, Dingbo Shi', Wuguo Deng ${ }^{5}$, Xinyue Cao ${ }^{6}$, Chong \\ Zhao $^{1,3}$ and Conghua Xie ${ }^{1}$ \\ ${ }^{1}$ Hubei Key Laboratory of Tumor Biological Behaviors, Department of Radiation and Medical Oncology, Zhongnan Hospital of \\ Wuhan University, Wuhan, China \\ ${ }^{2}$ Department of Radiation Oncology, Hubei Cancer Hospital, Wuhan, China \\ ${ }^{3}$ Department of Nasopharynx, Collaborative Innovation Center for Cancer Medical, State Key Laboratory of Oncology in \\ South China, Sun Yat-Sen University Cancer Center, Guangzhou, China \\ ${ }^{4}$ Department of Pathology, Hubei Cancer Hospital, Wuhan, China \\ ${ }^{5}$ Collaborative Innovation Center for Cancer Medical, State Key Laboratory of Oncology in South China, Sun Yat-Sen University \\ Cancer Center, Guangzhou, China \\ ${ }^{6}$ Department of Biological Repositories, Zhongnan Hospital of Wuhan University, Wuhan, China \\ *These authors contributed equally to this work \\ Correspondence to: Chong Zhao, email: zhaochong@sysucc.org.cn \\ Conghua Xie, email: chxie_65@whu.edu.cn
}

Keywords: PD-L1, PD-1, HIF- 1 $\alpha$, nasopharyngeal carcinoma, recurrent

Received: January 04, $2017 \quad$ Accepted: March 31, $2017 \quad$ Published: April 19, 2017

Copyright: Zhou et al. This is an open-access article distributed under the terms of the Creative Commons Attribution License 3.0 ( CC BY 3.0), which permits unrestricted use, distribution, and reproduction in any medium, provided the original author and source are credited.

\section{ABSTRACT}

The expression of Programmed death-1 (PD-1) / programmed death-ligand 1 (PD-L1) has been reported to be reliable prognostic factors in various malignances including primary nasopharyngeal carcinoma (NPC). However, the exact role of PD-1/ PD-L1 in recurrent NPC remains unclear. In this study, we aimed to investigate the relationship between the expression of PD-1 / PD-L1 and the clinical-pathology as well the outcomes of recurrent NPC patients $(n=132)$. The expression of PD-1 and PD-L1 was measured by immunohistochemistry staining. The relationship between PD-1 / PD-L1 and factors involved in clinic-pathology and outcomes of patients with NPC was assessed by correlation analysis. To further explore the association between PD-L1 and anemia, immunofluorescence analysis was performed to investigate the correlation of PD-L1 with hypoxia inducible factor-1 $\alpha$ (HIF-1 $\alpha$ ). We observed that advanced $\mathbf{r T}$ classification and anemia status before salvage treatment was associated with high level of PD-L1 in recurrent NPC patients, and PD-L1 and was co-located with HIF-1 $\alpha$ in recurrent tumors by immunofluorescence analysis. Moreover, our result suggested that PD-L1 might be a negative indicator for recurrent NPC patients as well as age, rT classification, anemia and tumor necrosis at diagnose of recurrence. Taken together, our results revealed that PD-L1 might be a potential prognostic biomarker for recurrent NPC patients, and advanced re-stage, anemia might represent as candidate biomarkers for evaluating patients' response to anti-PD-1 / PD-L1treatment. However, further studies are needed to clarify the underlying mechanism of hypoxia in immunosuppression process induced by PD-1 / PD-L1 axis. 


\section{INTRODUCTION}

Nasopharyngeal carcinoma (NPC) is endemic in southern China, where annual incidence ranges from 20 to 50 cases per 100,000 [1]. The local control of NPC was significantly improved due to advances in radiotherapy and combined chemotherapy, however, approximately $10 \%$ patients with NPC develop local and regional recurrences $[2,3]$. Recurrent NPC has a unique profile of pathological and clinical features $[4,5]$. Traditional salvage treatments offer limited clinical benefits and often cause severe complications. Although several biomarkers were identified for evaluating the prognosis of recurrent NPC, the overall survival (OS) rate of patients is still not improved with 5 -year OS rate being only $30 \%[6,7]$, making the management of recurrent NPC being a big challenge in clinic $[8,9]$. Therefore, seeking reliable prognostic markers as well as effective treatments are urgently required.

Programmed death-1 (PD-1 or CD279) is a coreceptor expressed predominantly by $\mathrm{T}$ cells [10]. Programmed death-ligand 1 (PD-L1 or CD274) engaged by PD-1 in cancer cells could inhibit the self-reactivation of $T$ cells and induce tumor immune tolerance [11]. The predictive value of PD-1 / PD-L1 has been recognized in multiple tumors [12-15], including primary NPC. However, the prognostic role of PD-1 and PD-L1 in NPC were controversy. For instance, our previous study [16] suggested that PD-L1 was correlated with worse outcome of primary NPC patients. Zhang et al [17] reported the similar negative correlation between PD-L1 and diseasefree survival of NPC. In contrast, Lee et al [18] delineated PD-L1 expression was positively correlated with longtime survival of NPC. Hsu et al [19] has shown that PD-1 on CD8 T cells predicted poor prognosis in 48 NPC patients. Interestingly, $\mathrm{PD}-1$ expression might not be a prognostic factor in NPC patients according our former study [16] and Zhang et al [17]. In addition to the conflicting findings in primary NPC, the prognostic value of PD-1 / PD-L1 in recurrent NPC has been rarely reported.

Therapies targeting the PD-1 / PD-L1 pathway show promising results in the treatment of cancers through promoting antitumor T-cell activity [20, 21]. There are also several ongoing clinical trials evaluating PD-1 / PDL1 checkpoint blockades in the treatment of NPC [22]. The overexpression of PD-1 / PD-L1 is presented as most effective predictive marker for evaluating patients' response to PD-1 / PD-L1 antibodies treatment [23, 24]. However, tumor microenvironment such as tumor hypoxia [25] might also account for the heterogeneous responses during immunotherapies. A previous study [26] demonstrated that PD-L1 is a direct target of hypoxia-induced factor- $1 \alpha$ (HIF$1 \alpha)$ and PD-L1 expression was up-regulated under hypoxia. In addition, blockage of PD-L1 enhanced myeloid-derived suppressor cells-mediated $\mathrm{T}$ cell activation, suggesting simultaneous blockage of PD-L1 and HIF- $1 \alpha$ might represent a novel approach for cancer immunotherapy.
In this study, we aimed to investigate the prognostic role of PD-1 and PD-L1 in a cohort of recurrent NPC patients $(n=132)$. In addition, we also evaluated the correlation between PD-1 / PD-L1 expression and clinicpathological variables as well as potential related peripheral blood biomarkers, such as hemoglobin (HB) level. Our study revealed that PD-L1 might be a potential prognostic biomarker for recurrent NPC patients, and advanced restage, anemia might represent as candidate biomarkers for evaluating patients' response to anti PD-1 / PD-L1-treatment.

\section{RESULTS}

\section{General information and survival outcomes}

All the biopsy samples of recurrent tumor $(\mathrm{n}=132)$ were collected. The samples were fixed by formalin and embedded into paraffin using a tissue processor. Among the 132 patients enrolled, there were 106 males and 26 females. The median time to recurrence after the initial treatment was 33.3 months (ranges from 6.6 to 190.2 months). The median age at recurrence was 46-year-old (ranges from 28 to 69 years). According to the $7^{\text {th }}$ edition of the UICC / AJCC system, 101 patients $(76.5 \%)$ were at III-VI stage and 32 patients (24.2\%) were lymph node positive simultaneously. Pathology proven recurrent undifferentiated non-keratinizing carcinoma (World Health Organization [WHO] III) and differentiated nonkeratinizing carcinoma (WHO II) were diagnosed in 125 and 7 patients, respectively. The median HB level before salvage therapy was $139.0 \mathrm{~g} / \mathrm{l}$ (ranges from 101.0 to $171.0 \mathrm{~g} / \mathrm{l})$ for all patients, among which 32 patients were anemic (HB $<130 \mathrm{~g} / \mathrm{l}$ in men; $<120 \mathrm{~g} / \mathrm{l}$ in women). Tumor necrosis before retreatment presented in 49 out of 132 (37.1\%) patients by pathologic diagnosis. Representative tumor necrosis with PD-L1 staining in recurrent NPC were shown in Figure 1. The average of follow-up time was 38.6 months (ranges from 3.5 to 182.1 months).

A total of 87 out of $132(65.9 \%)$ patients died during follow-ups. Of these, 35 out of 87 (40.2\%) died due to severe adverse effects, including 16 out of 35 (45.7 $\%)$ from mucosa necrosis or massive hemorrhage, 19 out of $35(54.3 \%)$ from other radiation-related injuries. In addition, 29 out of $87(33.3 \%)$ patients died due to local-regional failures and 16 out of $87(18.4 \%)$ due to distant failures. Other causes responsible for 2 out of 87 $(2.3 \%)$ deaths included 1 cases of cardiac disease, 1 case of digestive diseases. 5 out of 87 (5.7\%) patients died due to unknown reasons. The 3-year OS rates were $56.1 \%$ in the 132 recurrent patients. Characteristics of the enrolled patients were summarized in Table 1 .

\section{Clinic-pathologic correlations}

PD-1 positive immune cells presented in 50 of 132 patients $(37.9 \%)$ in a scattered manner, and PD-L1 staining was detectable in 128 tumors $(97.0 \%)$, which 
was mainly located at the membrane or in the cytoplasm region (or both) in the tumor cells. PD-L1 was also observed to be stained in tumor-infiltration lymphocytes (TILs) dispersedly. According to ROC curve analysis for OS, the optimal cut-off value of H-score was 190 (AUC: 0.702, sensitivity: 0.759, specificity: 0.511 ) for PD-L1. The staining of PD-L1 were considered as high level (88 cases) if $\mathrm{H}$-score $\geq 190$. Representative staining of PD-L1 and PD-1 in recurrent NPC were shown in Figure 1-2. Regarding the mortality of patients with high level of PD-L1 staining, there were 66 patients with high level of PD-L1 staining died during the follow-ups. Of these, 38 out of 66 patients died due to disease progression with 26 cases from local-regional failures and 12 cases from distant failures, another 28 out of 66 deaths were due to severe adverse effects, the cause of deaths were found to be significantly correlated with PD-L1 level $(\mathrm{P}=0.024$, Table 1). In this study, high level of PD-L1 staining was significantly correlated with clinical stage, rT classification of recurrent tumor and anemia at diagnose of recurrence. However, no correlation of high PD-L1 level with tumor necrosis, gender or time to recurrence was observed. No clinic-pathological parameters were found related to PD-1 positivity. Detailed data were summarized in Table 1.

\section{Correlation between PD-1 / PD-L1 expressions and anemia at recurrence}

Pre-retreatment hematologic biomarkers such as neutrophils, lymphocytes, $\mathrm{HB}$ and platelets were measured to identify the potential factors related to PD-1 / PD-L1. Our result showed that no significance (N. S.) between PD-1 positivity and HB level was observed in this cohort of recurrent patients (Table 1, Figure 3A). However, anemia status at diagnoses of recurrence was negatively correlated with $\mathrm{PD}-\mathrm{L} 1$ staining $(\mathrm{P}=0.044$, Table 1). Consistently, HB level was significantly reduced in patients with high level of $\mathrm{PD}-\mathrm{L} 1$ staining $(\mathrm{P}=0.029$, Figure 3B).

We have also compared PD-1 / PD-L1 expression with numbers of neutrophils, lymphocytes, platelets and neutrophils / lymphocytes ratio before salvage therapy
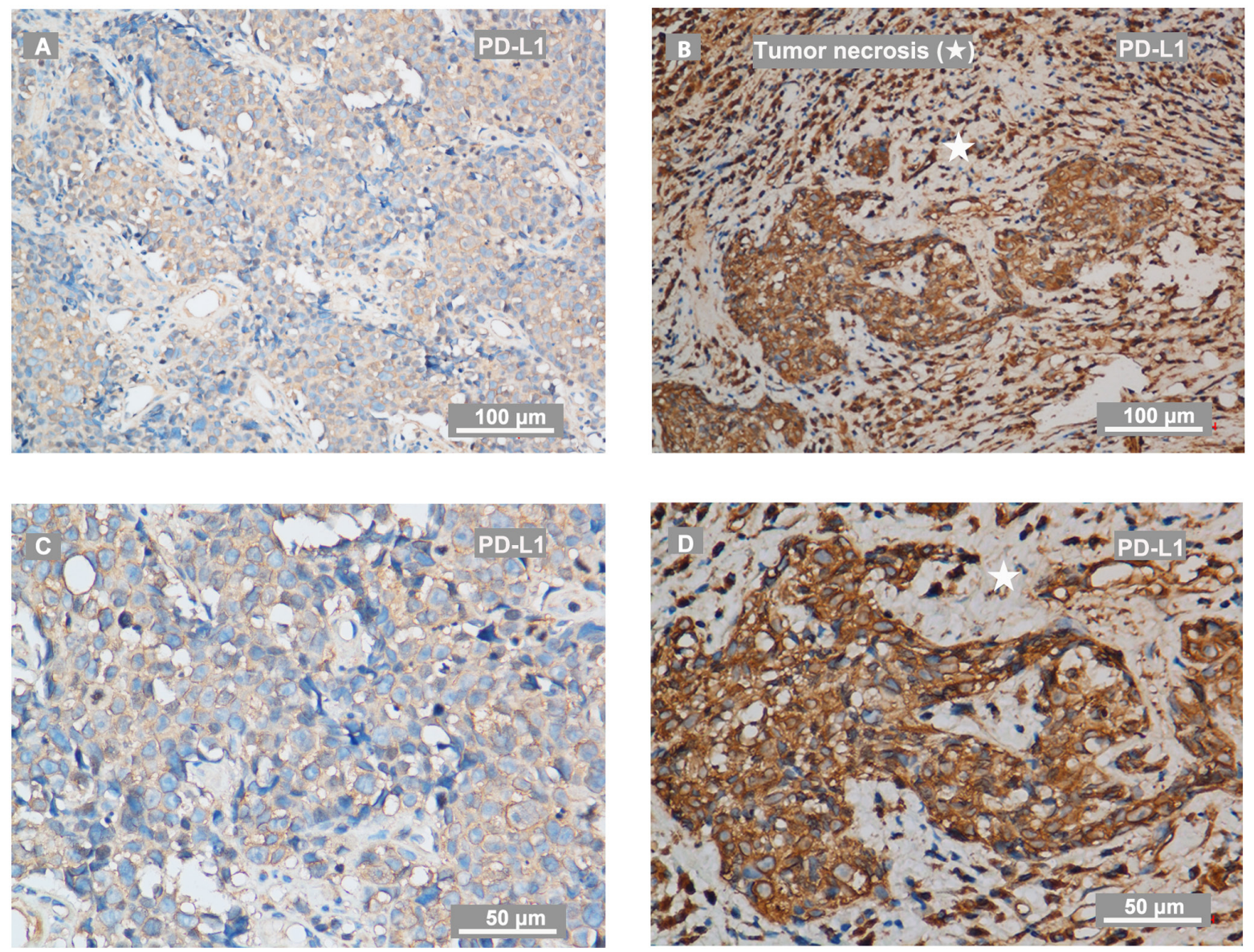

Figure 1: Representative IHC staining of PD-L1 and tumor necrosis on recurrent NPC-biopsies. (A, C) PD-L1 staining on biopsy from a patient with recurrent NPC evaluated as low level of PD-L1. The IHC photos were taken by a phase-contrast microscopy and exhibited as low (A) and high magnification (C). (B, D) PD-L1 staining on biopsy from a patient with recurrent NPC evaluated as high level of PD-L1. The IHC photos were exhibited as low (B) and high magnification (D). PD-L1 expressed in tumor-infiltration lymphocytes in a scattered manner. The scale bars for (A-B) are $100 \mu \mathrm{m}$, for $(\mathbf{C}-\mathbf{D})$ are $50 \mu \mathrm{m}$. $\star$ indicates tumor necrosis. 
Table 1: Clinic-pathologic variables and immune-activity status of PD-1 and PD-L1 in 132 patients with recurrent NPC

\begin{tabular}{|c|c|c|c|c|c|c|}
\hline Variables & & $\mathbf{n}$ & $\begin{array}{c}\text { PD-1 } \\
\text { positive }\end{array}$ & $P_{\text {value }}{ }^{1}$ & $\begin{array}{l}\text { PD-L1 } \\
\text { High }\end{array}$ & P value $^{1}$ \\
\hline \multirow[t]{2}{*}{ Gender } & Male & 106 & $41(38.7 \%)$ & 0.702 & $68(64.2 \%)$ & 0.216 \\
\hline & Female & 26 & $9(34.6 \%)$ & & $20(76.9 \%)$ & \\
\hline \multirow[t]{2}{*}{ Age at recurrence (years) } & $\leq 50$ & 87 & $32(36.8 \%)$ & 0.718 & $58(66.7 \%)$ & 1.000 \\
\hline & $>50$ & 45 & $18(40.0 \%)$ & & $30(66.7 \%)$ & \\
\hline \multirow[t]{2}{*}{ Time to recurrence (months) } & $\leq 24$ & 44 & $13(29.5 \%)$ & 0.163 & $26(59.1 \%)$ & 0.192 \\
\hline & $>24$ & 88 & $37(42.0 \%)$ & & $62(70.5 \%)$ & \\
\hline \multirow[t]{2}{*}{ Smoking status } & Smoker or ex-smoker & 52 & $22(42.3 \%)$ & 0.398 & $34(65.4 \%)$ & 0.801 \\
\hline & Non-smoker & 80 & $28(35.0 \%)$ & & $54(67.5 \%)$ & \\
\hline \multirow[t]{2}{*}{ Family history } & Yes & 28 & $13(46.4 \%)$ & 0.293 & $17(60.7 \%)$ & 0.452 \\
\hline & No & 104 & $37(35.6 \%)$ & & $71(68.3 \%)$ & \\
\hline \multirow[t]{2}{*}{ Histotype classification (WHO) } & II & 7 & $1(14.3 \%)$ & 0.186 & $5(71.4 \%)$ & 0.784 \\
\hline & III & 125 & $49(39.2 \%)$ & & $83(66.4 \%)$ & \\
\hline \multirow[t]{2}{*}{ r-T classification } & rT1-2 & 31 & $14(45.2 \%)$ & 0.339 & $13(41.9 \%)$ & $0.001 * *$ \\
\hline & rT3-4 & 101 & $36(35.6 \%)$ & & $75(74.3 \%)$ & \\
\hline \multirow[t]{2}{*}{ r-N category } & N0 & 100 & $37(37.0 \%)$ & 0.713 & $66(66.0 \%)$ & 0.774 \\
\hline & N1-3 & 32 & $13(40.6 \%)$ & & $22(68.8 \%)$ & \\
\hline \multirow[t]{2}{*}{$\begin{array}{l}\text { Clinical stage of recurrent } \\
\text { tumour }^{2}\end{array}$} & I-II & 31 & $14(45.2 \%)$ & 0.339 & $13(41.9 \%)$ & $0.001 * *$ \\
\hline & III-IV & 101 & $36(35.6 \%)$ & & $75(74.3 \%)$ & \\
\hline \multirow[t]{2}{*}{$\begin{array}{l}\text { Tumor necrosis before } \\
\text { retreatment }\end{array}$} & Yes & 49 & $18(36.7 \%)$ & 0.835 & $34(69.4 \%)$ & 0.610 \\
\hline & No & 83 & $32(38.6 \%)$ & & $54(65.1 \%)$ & \\
\hline \multirow[t]{2}{*}{ HB levels at local recurrence } & Anemia $^{3}$ & 32 & $11(34.4 \%)$ & 0.639 & $26(81.3 \%)$ & $0.044 \%$ \\
\hline & No anemia & 100 & $39(39.0 \%)$ & & $62(62.0 \%)$ & \\
\hline \multirow[t]{4}{*}{ Salvage treatments } & $\mathrm{RT}^{4}$ only & 43 & $20(46.5 \%)$ & 0.651 & $27(62.8 \%)$ & 0.111 \\
\hline & $\mathrm{RT}^{4}$ with $\mathrm{CT}^{5}$ & 80 & $31(38.8 \%)$ & & $58(72.5 \%)$ & \\
\hline & Surgery $^{6}$ & 6 & $2(33.3 \%)$ & & $2(33.3 \%)$ & \\
\hline & $\begin{array}{l}\text { Radiofrequency } \\
\text { ablation }^{6}\end{array}$ & 3 & $2(66.7 \%)$ & & $1(33.3 \%)$ & \\
\hline \multirow[t]{4}{*}{ Cause of deaths } & Disease progression & 45 & $19(42.2 \%)$ & 0.794 & $36(80.0 \%)$ & $0.024 *$ \\
\hline & Other disease & 2 & $1(50.0 \%)$ & & $1(50.0 \%)$ & \\
\hline & Severe adverse effects & 35 & $12(34.3 \%)$ & & $26(74.3 \%)$ & \\
\hline & Unknown & 5 & $3(60.0 \%)$ & & $3(60.0 \%)$ & \\
\hline
\end{tabular}

${ }^{1}$ According to the $7^{\text {th }}$ Edition of the AJCC / UICC Staging System for Nasopharyngeal Cancer. ${ }^{2}$ Anemia was defined according to World Health Organization criteria as hemoglobin $<130 \mathrm{~g} / 1$ in men and $<120 \mathrm{~g} / 1$ in women. ${ }^{3}$ Radiotherapy (RT) were proscribed to 114 patients by IMRT, 6 patients with conventional radiotherapy, 2 with stereotactic radiotherapy and 1 with brachytherapy. ${ }^{4}$ Combined chemotherapy (CT) were administered to 80 patients with RT. ${ }^{5}$ Induction chemotherapy were used in 2 out of 6 patients who received salvage surgery and 1 out of 3 patients treated with radiofrequency ablation. 

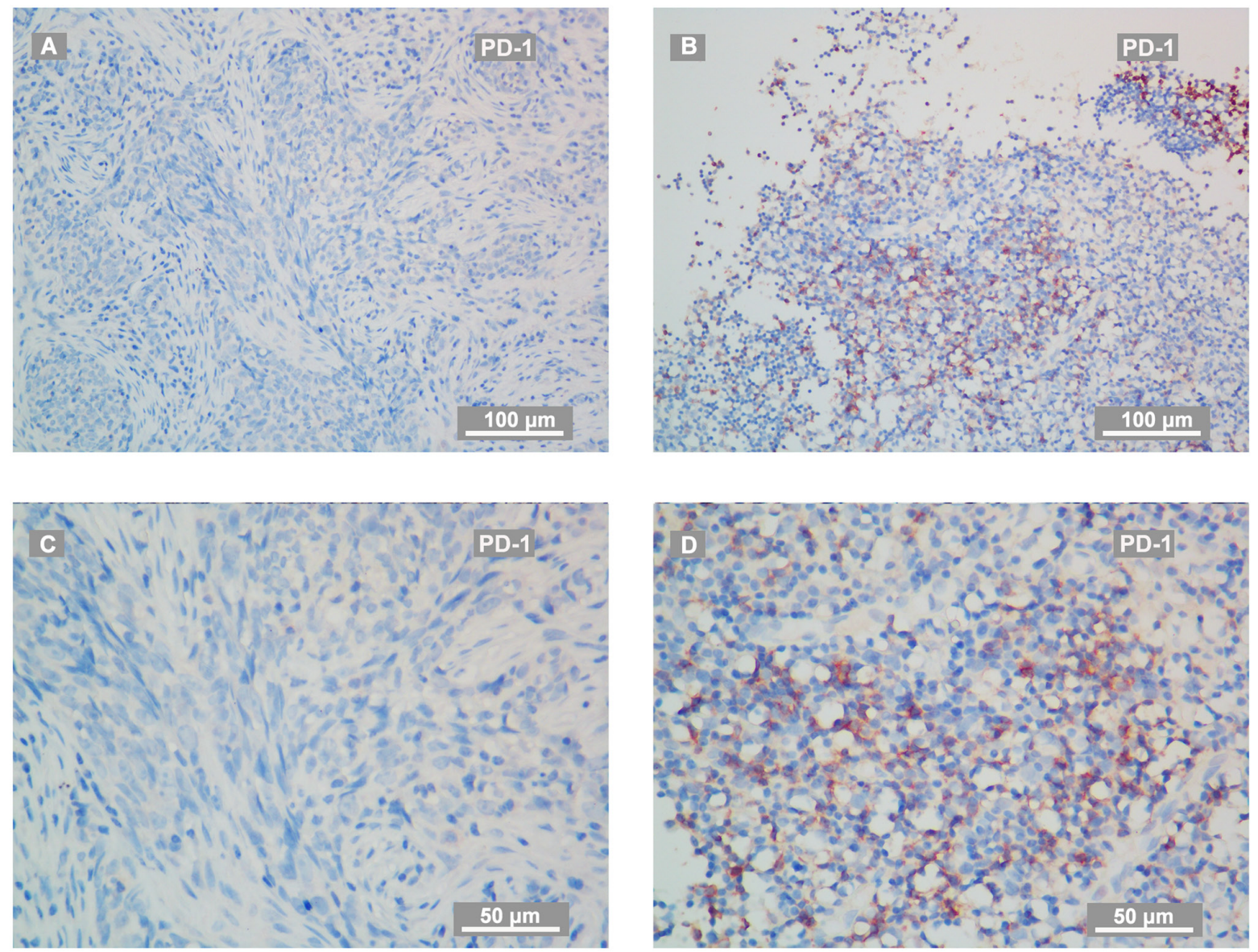

Figure 2: Representative IHC staining of PD-1 on recurrent NPC-biopsies. (A, C) PD-1 staining on biopsy from a patient with recurrent NPC evaluated as PD-1 negative. The IHC photos were taken by a phase-contrast microscopy and exhibited as low (A) and high magnification (C). (B, D) PD-1 staining on biopsy from a patient with recurrent NPC evaluated as PD-1 positive. The IHC photos were exhibited as low (B) and high magnification (D). The scale bars for (A-B) are $100 \mu \mathrm{m}$, for (C-D) are $50 \mu \mathrm{m}$. PD-1+ cells were tumorinfiltration lymphocytes (TILs).
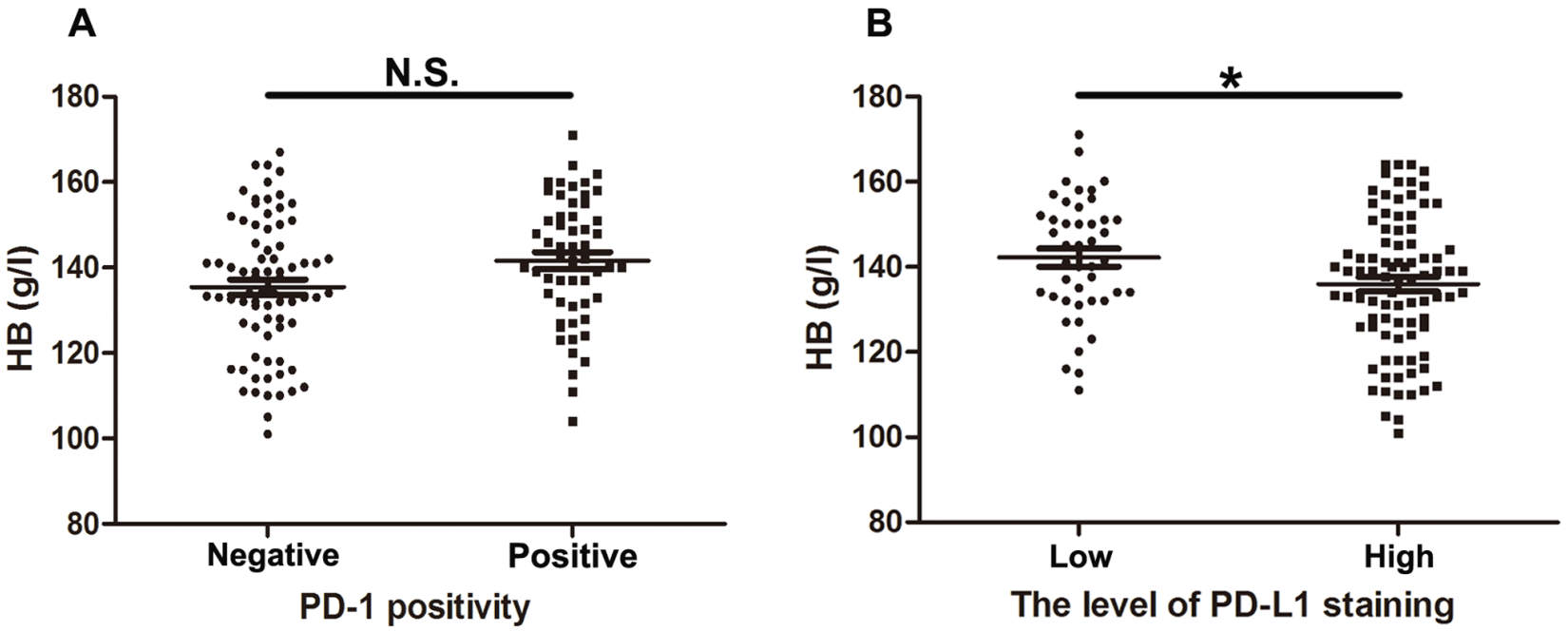

Figure 3: Hemoglobin in 132 NPC patients with different PD-1 / PD-L1 level. (A) Statistical analysis suggested no significant (N. S.) difference of HB level in recurrent patients with positive or negative PD-1. (B) Statistical analysis revealed significant decreased $\mathrm{HB}$ level in recurrent patients with high level of PD-L1. T-test was used to evaluate the association of PD1/PD-L1 with HB after normality tests and homogeneity of variance test. $* \mathrm{P}<0.05$. 
in this exploratory analysis, and no significantly relevant factors were identified (Supplementary Figure 1).

\section{Co-location of PD-L1 and hypoxia \\ inducible factor-1 $\alpha$ (HIF-1 $\alpha)$ by double immunofluorescence analysis}

Hypoxia-inducible factors (HIFs) have been demonstrated to play an important role in the regulation of erythropoiesis by coordinating a series of graded hypoxic responses [27] and stabilization of HIFs is represented as a novel therapeutic approach for the treatment of anemia [28]. Considering the closely association of HIF with anemia, immunofluorescence staining were performed to investigate the correlation of PD-L1 with anemia (represented as HIF-1 $\alpha$ activity) using 30 recurrent tumors. PD-L1 staining was detectable in 28 cases of recurrent tumors with different extent, which was mainly distributed in the cytoplasm region of the tumor cells. HIF-1 $\alpha$ staining was detectable in all 30 tumors, and was found to be colocated with PD-L1 staining regardless of the staining intensity or extent. Representative staining of PD-L1 and HIF- $1 \alpha$ in recurrent NPC by immunofluorescence was shown in Figure 4A-4L. Pearson correlation coefficient of merged staining with HIF-1 $\alpha$ and PD-L1 in Figure 4C, Figure 4F, Figure 4I, and Figure 4L were 0.94, 0.93, 0.76 and 0.93 , respectively.
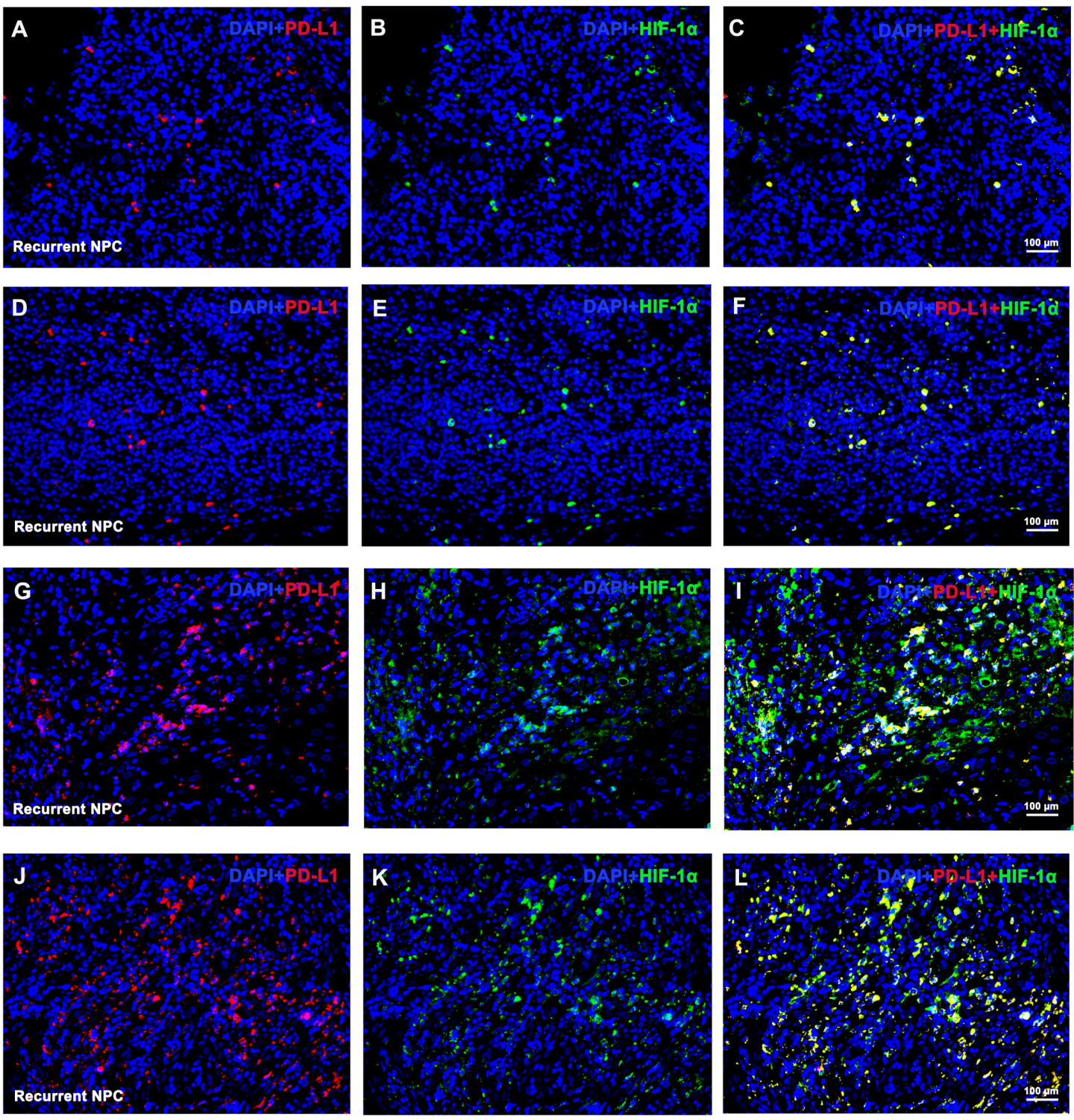

Figure 4: Representative double immunofluorescence staining of PD-L1 and HIF-1a on recurrent NPC-biopsies. Representative immunofluorescence staining for PD-L1 (red) and HIF-1 $\alpha$ (green) were co-located in space from 4 patients with recurrent NPC. 2 cases with relatively lower HIF-1 $\alpha$ level (A-C and D-F) and 2 with relatively higher HIF-1 $\alpha$ level cases (G-I and J-L) were selected as representative staining. Nuclear was stained by DAPI (blue). 


\section{Prognostic values in recurrent NPC}

To evaluate the prognostic values PD-L1 expression in recurrent NPC patients, we performed long rank tests in Kaplan-Meier survival analysis. In 132 patients with recurrent NPC, high expression of PD-L1 was correlated with significantly shorter $\mathrm{OS}(\mathrm{P}=0.001$, Figure $5 \mathrm{~A})$. Factors significantly correlated with OS by univariate analyses were age at recurrence $(\mathrm{P}=0.043$, Figure 5B), the level of PD-L1 staining $(\mathrm{P}=0.001$, Figure 5A), T-stage of recurrence $(\mathrm{P} \leq 0.001$, Figure $5 \mathrm{C})$, pre-retreatment anemia $(\mathrm{P}=0.007$, Figure $5 \mathrm{D})$ and tumor necrosis $(\mathrm{P}=$ 0.026 , Figure $5 \mathrm{E})$. However, PD-1 positivity on TILs was not significantly correlated with OS $(\mathrm{P}=0.950$, Table 2 ). Further analysis by dividing patients into 4 groups regarding PD-L1 level and tumor necrosis showed that patients with both tumor necrosis and high level of PD-L1 staining in recurrent NPC had the worst survival outcomes compared with patients in other groups $(\mathrm{P}=0.001$, Figure $5 \mathrm{~F})$. In the multivariate analysis, significantly negative prognostic factors were identified, including age $>50$ years, high level of PD-L1 staining, recurrent T3-4 stage and tumor necrosis before salvage treatment (Table 3). Moreover, patients with high level of PD-L1 might have higher risk of death $(95 \% \mathrm{CI}, 0.315-0.888, \mathrm{P}=0.016$, Table 3) in this cohort of 132 recurrent NPC patients.

\section{DISCUSSION}

The immunosuppression effect of PD-1 / PD-L1 and its prognostic value in various cancers are currently a research hotspot [25]. PD-L1 inhibits T cell-mediated antitumor immunity after engagement by PD-1 on TILs [29]. Recent studies revealed that PD-L1 expression on tumor cells might predict poor prognosis in most epithelial-originated cancers [30], suggesting an effect of PD-L1 on inducing tumor progression through regulation of antitumor immunity [31]. Recurrent NPC has unique biological characteristics and poor prognosis after salvage treatment. However, the prognostic significance of PD-L1 as well as PD-1 in the recurrent NPC has not been clarified yet.

We collected biopsies of recurrent tumor and clinical data from 132 recurrent NPC patients, to analyze the expression profiles of PD-L1 / PD-1 and related factors. Our results revealed rT classification was positively correlated with the level of PD-L1 staining, indicating that the aggressive behavior of recurrent NPC with advanced stage could be partially related to immune escape induced by PD-L1. Correspondingly, patients with high level of PD-L1 had a significantly reduced overall survival (Figure 5A), which was in consist with our previous study [16] and study conducted by Zhang et al [17] regarding primary NPC, indicating that PD-L1 might be a potential prognostic biomarker for both primary and recurrent NPC patients. Furthermore, we analyzed the correlation between positivity of PD-1 and survival outcomes of recurrent NPC patients and found no significance available (Table 1). In primary NPC, a previous study suggested a poor prognosis of OS in primary NPC patients with PD1-positive CD8 T cells (total $\mathrm{n}=46$ ) [19]. However, a larger cohort (total $n=139$ ) demonstrated that the impact of PD-1 was not significant [17]. The prognostic value of PD-1 was largely undefined in NPC, one possible illustration might be that PD-1 expression in TILs was usually dynamically changed according to the immune status [32], and the prognostic role of PD-1 in recurrent NPC as well as in primary tumors still needs to be further clarified.

Identification of potential factors will aid in stratifying the response to anti-PD-1 blockades. Our study showed high level of PD-L1 staining was significantly correlated with anemia status at diagnose of recurrence (Table 1). Patients with high level of PD-L1 staining had significantly reduced HB levels (Figure 3B). Previous studies confirmed low baseline HB level is an adverse prognostic factor in patients with locally advanced head and neck squamous cell carcinoma [33] and NPC [34], by inducing treatment resistance via enhancing tumor hypoxia [35]. Additionally, this exploratory study revealed the co-location of PD-L1 with HIF-1 $\alpha$, the latter of which was known as a central element in the response to hypoxia [36]. Recent studies confirmed that hypoxia via HIF- $1 \alpha$ might selectively up-regulate PDL1 on immunosuppressive cells [26] and solid tumors such as pulmonary pleomorphic carcinoma [37], and oral squamous cell carcinoma [38], which contributed to the evidence for hypoxia induced immune deficiency by PD-1 / PD-L1 axis [39]. Following studies confirmed that upregulation of PD-L1 expression induced by hypoxia was mediated by STAT3 signaling pathway in anaplastic lymphoma kinase (ALK) positive pulmonary adenocarcinomas [40]. While the potential molecular mechanism behind PD-L1 and hypoxia in recurrent NPC remains unclear and requires further investigation.

Our study revealed the prognostic role of age at recurrence, PD-L1 level, anemia, tumor necrosis and rT classification in 132 recurrent NPC patients by log-rank test. Consistently, age at recurrence, rT classification, volume of recurrent tumor, response of re-radiotherapy, as well as significant complications were reported to have prognostic role in recurrent NPC patients as demonstrated by previous studies $[6,41]$. In this study, we showed that tumor necrosis was correlated with worse outcomes in recurrent NPC, which was consistent with previous works $[42,43]$. Notably, further analysis showed the patients with both tumor necrosis and high level of PD-L1 staining in recurrent NPC had worst survival outcomes, whereas, those patients with no tumor necrosis and low level of PD-L1 staining had better prognosis. In consist with our findings, a former study found that PD-L1 accompanied by HIF- $1 \alpha$ surrounding necrosis in oral squamous cell 
A
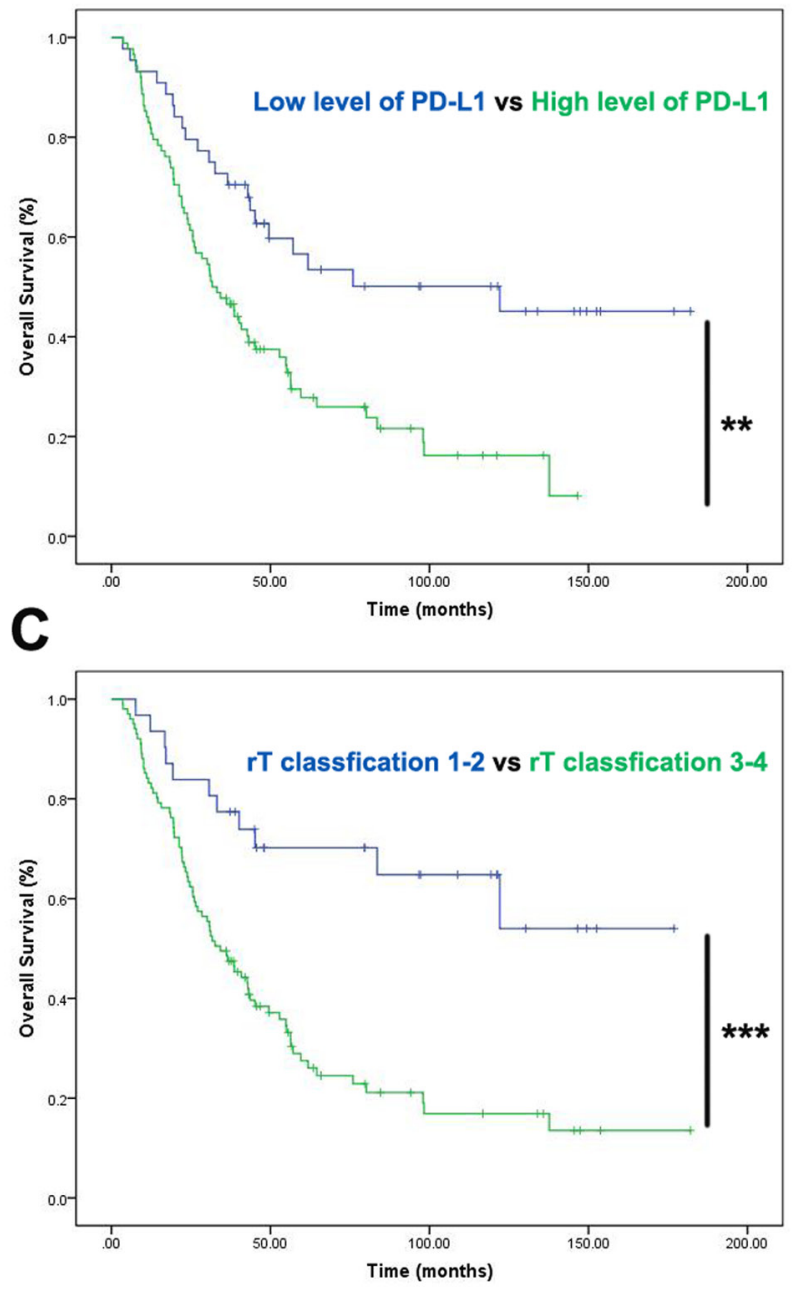

E

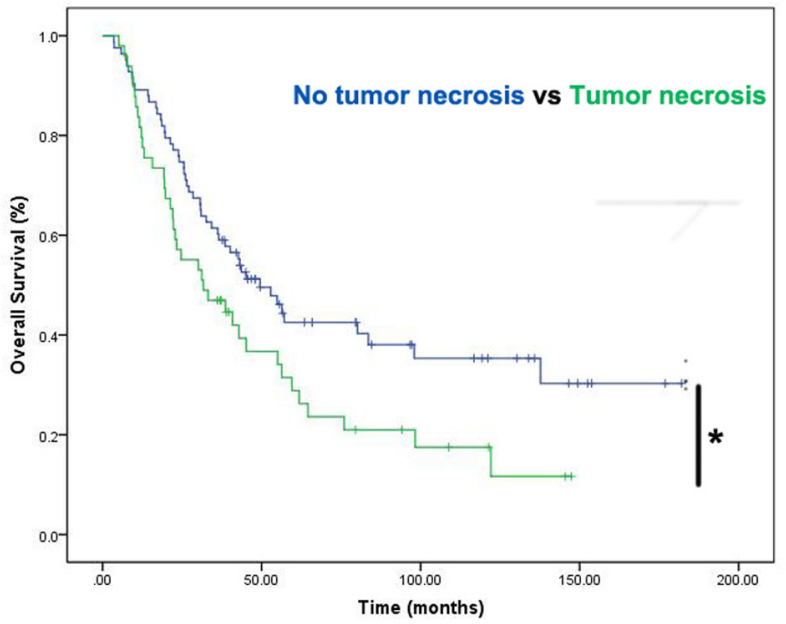

B
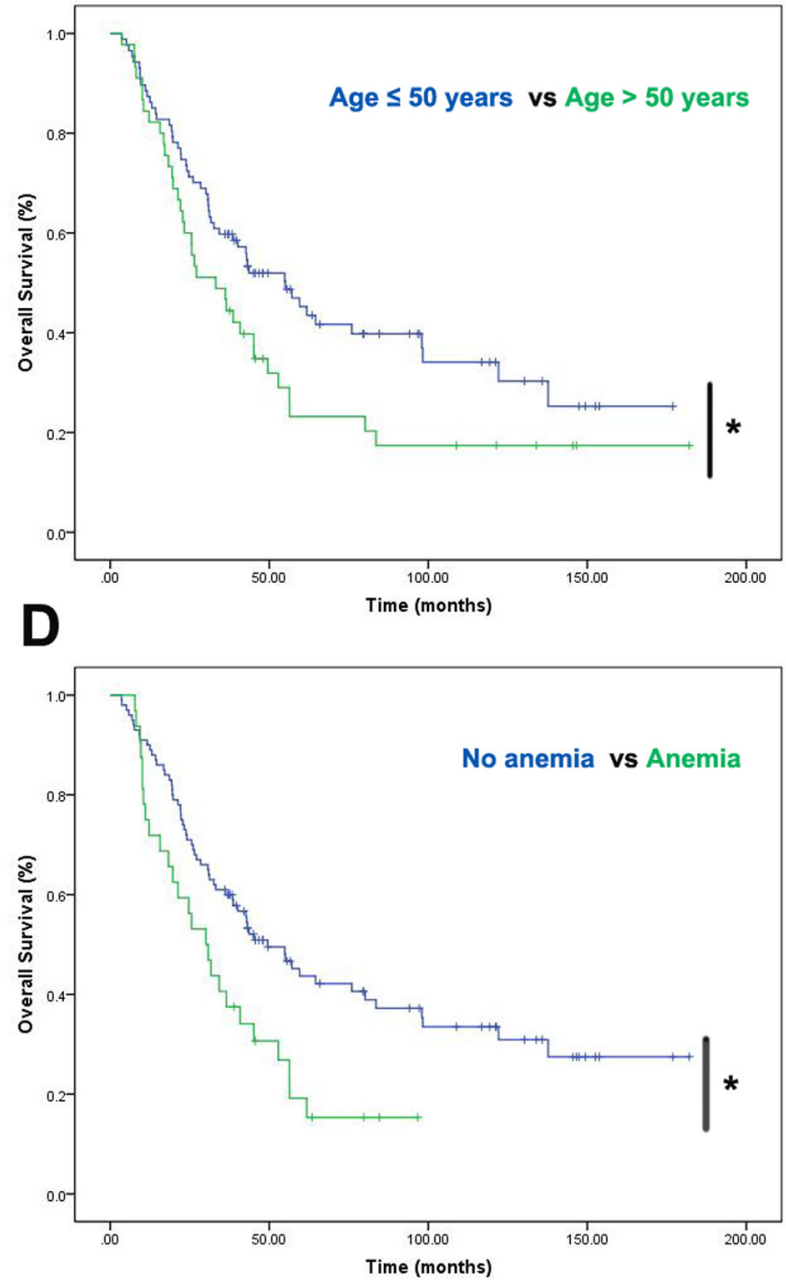

$\mathbf{F}$

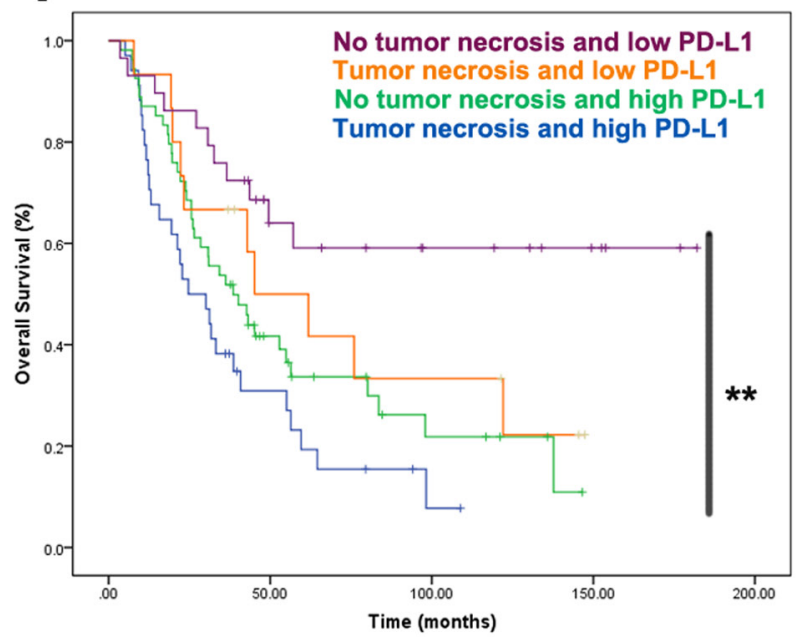

Figure 5: Prognostic factors of the 132 patients with recurrent NPC by Kaplan-Meier survival analysis. (A-E) Results revealed significant links between overall survival and high level of PD-L1 (A), age (B), rT classification (C), anemia (D) as well as tumor necrosis (E). The cut-off points of PD-L1 were generated from ROC. (F) When considered as combined factors, high level of PD-L1 staining accompanied with tumor necrosis predicted the worst survival outcomes comparing with the other groups in recurrent NPC patients. Survival analysis was depicted by Kaplan-Meier method. Univariate analysis were performed with log-rank test. * $\mathrm{P}<0.05$, ** $\mathrm{P}<0.01$. 
Table 2: Univariate analysis of prognostic factors involved in OS with recurrent NPC

\begin{tabular}{|c|c|c|c|c|c|c|}
\hline \multirow{2}{*}{ Variables } & & \multirow{2}{*}{$\begin{array}{c}\text { Cases } \\
(n=132)\end{array}$} & \multicolumn{4}{|c|}{ Overall Survive rate } \\
\hline & & & 3-years & 5-years & $\mathbf{X}^{2}$ & P value $^{1}$ \\
\hline \multirow[t]{2}{*}{ Gender } & Male & 106 & $60(56.6 \%)$ & $27(25.5 \%)$ & 0.009 & 0.925 \\
\hline & Female & 26 & $14(53.8 \%)$ & $7(26.9 \%)$ & & \\
\hline \multirow[t]{2}{*}{ Age at recurrence (years) } & $\leq 50$ & 87 & $52(59.8 \%)$ & $26(29.9 \%)$ & 4.077 & $0.043 *$ \\
\hline & $>50$ & 45 & $22(48.9 \%)$ & $8(17.8 \%)$ & & \\
\hline \multirow[t]{2}{*}{ Smoking status } & Smoker or ex-smoker & 52 & $26(50.0 \%)$ & $12(23.1 \%)$ & 1.738 & 0.187 \\
\hline & Non-smoker & 80 & $48(60.0 \%)$ & $22(27.5 \%)$ & & \\
\hline \multirow[t]{2}{*}{ Family history } & Yes & 28 & $15(53.6 \%)$ & $9(32.1 \%)$ & 0.023 & 0.881 \\
\hline & No & 104 & $59(56.7 \%)$ & $25(24.0 \%)$ & & \\
\hline \multirow[t]{2}{*}{$\begin{array}{l}\text { Histotype classification } \\
\text { (WHO) }\end{array}$} & II & 7 & $4(57.1 \%)$ & $2(28.6 \%)$ & 0.770 & 0.380 \\
\hline & III & 125 & $70(56.0 \%)$ & $32(25.6 \%)$ & & \\
\hline \multirow[t]{2}{*}{ r-T classification } & rT1-2 & 31 & $24(77.4 \%)$ & $15(48.4 \%)$ & 14.698 & $0.000 * * *$ \\
\hline & rT3-4 & 101 & $50(49.5 \%)$ & $19(18.8 \%)$ & & \\
\hline \multirow[t]{2}{*}{$\mathrm{r}-\mathrm{N}$ category } & No & 100 & $57(57.0 \%)$ & $28(28.0 \%)$ & 0.177 & 0.674 \\
\hline & N1-3 & 32 & $17(53.1 \%)$ & $6(18.8 \%)$ & & \\
\hline \multirow[t]{2}{*}{$\begin{array}{l}\text { Clinical stage of recurrent } \\
\text { tumor }^{2}\end{array}$} & I-II & 31 & $24(77.4 \%)$ & $15(48.4 \%)$ & 14.698 & $0.000 * * *$ \\
\hline & III-IV & 101 & $50(49.5 \%)$ & $19(18.8 \%)$ & & \\
\hline \multirow[t]{2}{*}{$\begin{array}{l}\text { Tumor necrosis before } \\
\text { retreatment }\end{array}$} & Yes & 49 & $23(46.9 \%)$ & $11(22.4 \%)$ & 4.958 & $0.026 *$ \\
\hline & No & 83 & $51(61.4 \%)$ & $23(27.7 \%)$ & & \\
\hline \multirow[t]{2}{*}{$\begin{array}{l}\text { HB levels at local } \\
\text { recurrence }\end{array}$} & Anemia $^{3}$ & 32 & $13(40.6 \%)$ & $5(15.6 \%)$ & 7.200 & $0.007 *$ \\
\hline & No anemia & 100 & $61(61.0 \%)$ & $29(29.0 \%)$ & & \\
\hline \multirow[t]{2}{*}{$\begin{array}{l}\text { Time to recurrence } \\
\text { (months) }\end{array}$} & $\leq 24$ & 44 & $26(59.1 \%)$ & $8(18.2 \%)$ & 0.394 & 0.530 \\
\hline & $>24$ & 88 & $48(54.5 \%)$ & $26(29.5 \%)$ & & \\
\hline \multirow[t]{4}{*}{ Salvage treatments } & $\mathrm{RT}^{4}$ only & 43 & $24(55.8 \%)$ & $13(30.2 \%)$ & 2.486 & 0.478 \\
\hline & $\mathrm{RT}^{4}$ with $\mathrm{CT}^{5}$ & 80 & $43(53.8 \%)$ & $20(25.0 \%)$ & & \\
\hline & Surgery $^{6}$ & 6 & $5(83.3 \%)$ & $0(0.0 \%)$ & & \\
\hline & $\begin{array}{l}\text { Radiofrequency } \\
\text { ablation }^{6}\end{array}$ & 3 & $2(66.7 \%)$ & $1(33.3 \%)$ & & \\
\hline \multirow[t]{2}{*}{ PD-1 positivity } & Negative & 82 & $43(52.4 \%)$ & $21(25.6 \%)$ & 0.004 & 0.950 \\
\hline & Positive & 50 & $31(62.0 \%)$ & $13(26.0 \%)$ & & \\
\hline \multirow[t]{2}{*}{ PD-L1 level ${ }^{7}$} & Low & 43 & $32(74.4 \%)$ & $18(41.9 \%)$ & 11.701 & $0.001 * *$ \\
\hline & High & 96 & $42(43.8 \%)$ & $16(16.7 \%)$ & & \\
\hline
\end{tabular}

(Continued) 


\begin{tabular}{|c|c|c|c|c|c|c|}
\hline \multirow{2}{*}{ Variables } & & \multirow{2}{*}{$\begin{array}{c}\text { Cases } \\
(\mathrm{n}=132)\end{array}$} & \multicolumn{4}{|c|}{ Overall Survive rate } \\
\hline & & & 3-years & 5-years & $\mathrm{X}^{2}$ & Pvalue $^{1}$ \\
\hline \multirow[t]{4}{*}{$\begin{array}{l}\text { PD-L1 level and Tumor } \\
\text { necrosis }\end{array}$} & $\begin{array}{c}\text { No tumor necrosis and } \\
\text { low PD-L1 }\end{array}$ & 29 & $22(75.9 \%)$ & $12(41.4 \%)$ & 16.606 & $0.001 * *$ \\
\hline & $\begin{array}{l}\text { Tumor necrosis and } \\
\text { low PD-L1 }\end{array}$ & 15 & $10(66.7 \%)$ & $6(40.0 \%)$ & & \\
\hline & $\begin{array}{c}\text { No tumor necrosis and } \\
\text { high PD-L1 }\end{array}$ & 54 & $29(53.7 \%)$ & $11(20.4 \%)$ & & \\
\hline & $\begin{array}{c}\text { Tumor necrosis and } \\
\text { high PD-L1 }\end{array}$ & 34 & $13(38.2 \%)$ & $5(14.7 \%)$ & & \\
\hline
\end{tabular}

${ }^{1}$ Log-rank test. ${ }^{2}$ According to the $7^{\text {th }}$ Edition of the AJCC / UICC Staging System for Nasopharyngeal Cancer. ${ }^{3}$ Anemia was defined according to World Health Organization criteria as hemoglobin $<130 \mathrm{~g} / 1$ in men and $<120 \mathrm{~g} / \mathrm{l}$ in women. ${ }^{4}$ Radiotherapy (RT) were proscribed to 114 patients by IMRT, 6 patients with conventional radiotherapy, 2 with stereotactic radiotherapy and 1 with brachytherapy. ${ }^{5}$ Combined chemotherapy (CT) were administered to 80 patients with RT.

${ }^{6}$ Induction chemotherapy were used in 2 out of 6 patients who received salvage surgery and 1 out of 3 patients treated with radiofrequency ablation. ${ }^{7}$ Optimal cut-off point for PD-L1 H-score were determined by the receiver operating characteristic (ROC) curve for overall survival (OS). ${ }^{*} \mathrm{P}<0.05,{ }^{*} \mathrm{P}<0.01,{ }^{*} * * \mathrm{P}<0.001$.

Table 3: Multivariate analysis of prognostic factors involved in survival

\begin{tabular}{lccccc}
\hline Variables & Category & $\boldsymbol{\beta}$ & P value $^{\mathbf{1}}$ & Hazard ratio & $\begin{array}{c}\mathbf{9 5 \%} \text { confidence } \\
\text { interval }\end{array}$ \\
\hline $\begin{array}{l}\text { Age at recurrence } \\
\begin{array}{l}\text { Clinical stage of } \\
\text { recurrent tumour }\end{array}\end{array}$ & $>\mathbf{5 0}$ years vs $\leq \mathbf{5 0}$ years & -0.453 & $\mathbf{0 . 0 4 3}$ & 0.636 & $0.410-0.986$ \\
$\begin{array}{l}\text { Tumor necrosis before } \\
\text { retreatment }\end{array}$ & III-IV vs I-II & -0.988 & $\mathbf{0 . 0 0 3 * *}$ & 0.372 & $0.193-0.717$ \\
$\begin{array}{l}\text { HB levels at local } \\
\text { recurrence }\end{array}$ & Yes vs No & -0.437 & $\mathbf{0 . 0 4 5 *}$ & 0.646 & $0.422-0.989$ \\
$\begin{array}{l}\text { PD-L1 level } \\
\text { Anemia }\end{array}$ & High vs No anemia & -0.329 & 0.182 & 0.720 & $0.444-1.166$ \\
\hline
\end{tabular}

${ }^{1}$ Cox regression analysis. ${ }^{2}$ According to the $7^{\text {th }}$ Edition of the AJCC / UICC Staging System for Nasopharyngeal Cancer. ${ }^{3}$ Anemia was defined according to World Health Organization criteria as hemoglobin $<130 \mathrm{~g} / \mathrm{l}$ in men and $<120 \mathrm{~g} / \mathrm{l}$ in women. ${ }^{4}$ Optimal cut-off point for PD-L1 H-score were determined by the receiver operating characteristic (ROC) curve for overall survival (OS). $* \mathrm{P}<0.05, * * \mathrm{P}<0.01$.

carcinoma predicted a worse outcome [38]. The exact mechanism underlying the formation of tumor necrosis has yet to be fully elucidated. Pathologic changes might occur in the early stage of necrosis [43]. It has been reported that tumor necrosis often occurred in recurrent tumors due to hypoxia, hypovascularity and hypocellularity which were induced by the first course of radiotherapy [44]. In our study, anemia was not significantly correlated with tumor necrosis $(\mathrm{P}=0.186$, Chi square test), indicating the complex process besides anemia in forming a tumor necrosis. These findings from our study may have implications for the future design of randomized clinical trials regarding to the application of PD-1 / PD-L1 pathway blockades in the treatment of the recurrent NPC.

In conclusion, PD-L1 and PD-1 are widely expressed in recurrent NPC tissue. PD-L1 level, age at recurrence, re-stage and tumor necrosis were prognostic factors for recurrent NPC patients. In addition, Advanced re-stage and anemia might represent as candidate biomarkers for evaluating patients' response to antiPD-1 / PD-L1-treatment. Further studies with a larger size of samples involved are needed to confirm these observations. Moreover, more researches are warranted to identify the molecular mechanism of the interaction between hypoxia and PD-L1 in recurrent NPC.

\section{MATERIALS AND METHODS}

\section{Ethics statement}

All data collection and statistical analysis of this study were conducted in accordance with the Institutional 
Review Board of Sun Yat-Sen University Cancer Center. Informed consent was obtained from all subjects before their salvage treatment. Individual informed consent was waived based on the Ethical Board requirement for retrospective clinical studies. Patient records / information was anonymized and de-identified prior to analysis.

\section{Patients and samples}

A total of 179 patients with locally recurrent NPC from Sun Yat-Sen University Cancer Centre between January 2001 and March 2013 were enrolled. The cases were selected based on the following criteria: (1) local recurrence was histologically confirmed with available biopsy specimens; (2) complete clinical data; (3) nondistant metastatic simultaneously; (4) the main treatment of the initial course was radiotherapy; (5) no other malignant diseases; (6) Karnofsky score $\geq 70$; (7) received salvage treatment at Sun Yat-Sen University; (8) followup regularly. Patients with active hepatitis, diabetes, or who failed the follow-up requirements were excluded. Therefore, we obtained the remaining 132 cases qualified for this study. All patients with recurrent NPC were restaged according to the $7^{\text {th }}$ edition of the UICC /AJCC system. Formalin-fixed and paraffin-embedded (FFPE) blocks from fiberscope biopsy of NPC were retrieved from the Department of Pathology. The related clinical data of these patients were retrospectively collected from our database or hospital records, including gender, age, smoking status, family history, clinical stage, pre-therapy laboratory counts of neutrophils, lymphocytes, HB and platelets as well as follow-up records. Tumor necrosis was defined as the necrosis of tumor tissue at diagnose of recurrence and assessed pathologically by two experienced pathologists independently. Anemia was defined according to World Health Organization criteria as hemoglobin < $130 \mathrm{~g} / \mathrm{l}$ in men and $<120 \mathrm{~g} / \mathrm{l}$ in women. All patients were followed-up for at least 3 years.

\section{Salvage treatment}

According to the discretion of attending oncologists with the consideration of disease condition and the preference of patients, most of these recurrent patients $(114 / 132,86.4 \%)$ were given salvage IMRT, 6 patients were treated with conventional radiotherapy, 2 with stereotactic radiotherapy, 1 with brachytherapy and 3 radiofrequency ablation, salvage endoscopic nasopharyngectomy were performed for 6 patients according to the procedure previously reported by Chen MY et al [45]. The prescribed doses of IMRT to the recurrent tumors were 60-70 Gy in 27-35 fractions according to the institutional protocol delineated by Tian YM et al [41]. Cisplatin-based induction, concurrent or adjuvant chemotherapy with radiotherapy was administered to 78 patients with rT3-4 and / or bulky gross tumors. The groups included 29 patients with concurrent chemo-radiotherapy, 32 patients with induction chemotherapy followed by radiotherapy, 13 patients with induction and concurrent chemotherapy, 4 patients with adjuvant chemotherapy. Besides, 2 out of 6 patients who received salvage surgery and 1 out of 3 patients treated with radiofrequency ablation were administered with induction chemotherapy. 13 patients $(9.8 \%)$ received targeted agents (cetuximab, nimotuzumab or bevacizumab) combined with their other treatment schedules.

\section{Follow-up}

All patients were required to be followed-up every 3-4 months in the first 3 years, every 6 months for 2 additional years, and annually after their salvage treatment. Each follow-up visit included physical examination, indirect nasopharyngoscopy, MRI of the head-and-neck regions, $\mathrm{CT}$ of thoracic region and ultrasound or $\mathrm{CT}$ of the abdomen.

\section{FFPE}

The samples were fixed by $4 \%$ paraformaldehyde containing $2 \%$ sucrose in PBS at $4{ }^{\circ} \mathrm{C}$ for overnight and embedded into paraffin using a tissue processor (EG1150, Leica, Germany). FFPE sections (3 $\mu \mathrm{m}$ thick) were cut with a rotation microtome (RM2255, Leica, Germany).

\section{Immunohistochemistry staining}

Paraffin sections were dewaxed and rehydrated by alcohol series, and then were treated with $3 \% \mathrm{H}_{2} \mathrm{O}_{2}$ for $10 \mathrm{~min}$ at room temperature. The sections were steam heated for $2.5 \mathrm{~min}$ in ethylene diamine tetra acetic acid buffer $(\mathrm{PH}=8.0)$ to retrieve antigen. Subsequent staining was performed with a $50 \mathrm{~min}$ incubation period at $37^{\circ} \mathrm{C}$ with the monoclonal antibodies PD-1 and PD-L1 (Supplementary Table 1). The immunostaining of PD-1 and PD-L1 proteins was performed on two different slides. Tonsils tissue was used as a positive control. Immunoreaction was visualized by a Peroxidase / DAB kit (Cat. K5007, Dako, Denmark). Images were taken with a phase contrast microscope (Eclipse 80i, Nikon, Japan).

\section{Evaluation standard of IHC}

Percentages of PD-L1 positive tumor cells and PD-1 positive TILs and staining intensity were evaluated by two experienced pathologists independently who remained blind to clinical information by counting 20 sequential high-power fields (0.54 mm field diameter). Staining intensity was scored by the criteria: 0 as negative or trace, 1 as weak, 2 as moderate and 3 as high. The percentage of positive cells $(0-100 \%)$ was multiplied by the dominant intensity score of staining ranging from 
0 to 3 . Therefore, the overall semi-quantitative score, that is $\mathrm{H}$-score, was ranged from 0 to 300 (maximum value of 300 corresponding to $100 \%$ of tumor cells for PD-L1 or TILs for PD-1 staining with an overall staining intensity score of 3 ).

\section{Immunofluorescence staining}

Paraffin sections were rehydrated and steam heated to retrieve antigen under the same conditions as in IHC procedure, and blocked in normal goat serum for $30 \mathrm{~min}$ at room temperature. The sections were incubated with primary antibodies against PD-L1 and HIF-1 $\alpha$ (Supplementary Table 1), followed by addition of Alexa-Fluor -555 or Alexa-Fluor-488 labeled secondary antibodies (Supplementary Table 2) respectively in humidified atmosphere. Nuclei were labeled with DAPI in a concentration of $2 \mu \mathrm{g} / \mathrm{ml}$ (Supplementary Table 2). Sections were analyzed by a fluorescence microscope (Olympus, Cat. \#BX53). Immunofluorescence analysis was performed by two experienced pathologists independently. Pearson's correlation coefficient was performed by Image-Pro plus 6.0 to evaluate the colocation of PD-L1 with HIF-1 $\alpha .0$ indicates no significant correlation, -1.0 indicates complete negative correlation, and 1.0 indicates complete positive correlation.

\section{Statistical analyses}

Optimal cut-off point for PD-L1 H-score was determined by the area under the curve (AUC) of the receiver operating characteristic (ROC) curve at the highest positive likelihood ratio point for OS. PD-L1 expression was dichotomized into two groups (high and low), using a cut-off score of $\geq 190$. OS was measured from the diagnosis of local recurrence until confirmed death or the date of last follow-up through April 2016.

These statistical analyses were performed with SPSS 16.0. Measurement data were presented as mean \pm standard error (S.E.M) Chi square test was used to assess the level of PD-1 and PD-L1 correlated with various clinical parameters such as age, clinical stage, anemia and tumor necrosis before re-therapy. T-test was used to evaluate the association of PD1 / PD-L1 with $\mathrm{HB}$, neutrophil counts and platelet after normality test (Kolmogorov-Smirnov test). Nonparametric MannWhitney $U$ test was applied to evaluate the association of PD-1 / PD-L1 with lymphocyte count and neutrophil / lymphocyte ratio. Survival analysis was depicted by Kaplan-Meier method. Univariate analysis and multivariate analysis were performed with log-rank test and Cox regression analysis, respectively. $\mathrm{P}$ value $<0.05$ was used to denote statistical significant, and all reported $\mathrm{P}$ values were two sided. $\mathrm{P}$ values were marked as $* \mathrm{P}<$ $0.05, * * \mathrm{P}<0.01, * * * \mathrm{P}<0.001$, indicating different level of significance.

\section{Abbreviations}

PD-1, programmed death-1; PD-L1, programmed death-ligand 1; NPC, nasopharyngeal carcinoma; HIF$1 \alpha$, hypoxia inducible factor $1 \alpha$; OS, overall survival; IMRT, intensity modulated radiation therapy; HB, hemoglobin; UICC, Union for International Cancer Control; AJCC, American Joint Committee on Cancer; WHO, World Health Organization; TILs, tumor-infiltration lymphocytes; ROC, receiver operating characteristic; STAT3, signal transducer and activator of transcription 3; ALK, anaplastic lymphoma kinase; FFPE, formalinfixed and paraffin-embedded; MRI, Magnetic Resonance Imaging; CT, Computed Tomography; PET-CT, Positron Emission Tomography-Computed Tomography; IHC, Immunohistochemistry.

\section{ACKNOWLEDGMENTS}

We thank the Department of Nasopharyngeal Carcinoma from Sun Yat-Sen University Cancer Center for providing tissue samples. We also would like to thank Prof. Dr. Chuanhua Yu from School of Public Health, Wuhan University for suggestions to improve our statistical analysis.

\section{CONFLICTS OF INTEREST}

Competing financial interests: The authors declare no competing financial interest.

\section{FUNDING SUPPORT}

This study was supported in part by grants from the Zhongnan Hospital of Wuhan University Science, Technology and Innovation Seed Fund (grant number cxpy20160050) and the Natural Sciences Foundation of Hubei Province (grant number 2013CFA006). The funders had no role in study design, data collection and analysis, decision to publish, or preparation of the manuscript.

\section{REFERENCES}

1. Luo J, Chia KS, Chia SE, Reilly M, Tan CS, Ye W. Secular trends of nasopharyngeal carcinoma incidence in Singapore, Hong Kong and Los Angeles Chinese populations, 19731997. Eur J Epidemiol. 2007; 22:513-21.

2. Sun X, Su S, Chen C, Han F, Zhao C, Xiao W, Deng $\mathrm{X}$, Huang S, Lin C, Lu T. Long-term outcomes of intensity-modulated radiotherapy for 868 patients with nasopharyngeal carcinoma: an analysis of survival and treatment toxicities. Radiother Oncol. 2014; 110:398-403.

3. Zhang B, Mo Z, Du W, Wang Y, Liu L, Wei Y. Intensitymodulated radiation therapy versus $2 \mathrm{D}-\mathrm{RT}$ or $3 \mathrm{D}-\mathrm{CRT}$ for 
the treatment of nasopharyngeal carcinoma: A systematic review and meta-analysis. Oral Oncol. 2015; 51:1041-46.

4. Li JX, Lu TX, Huang Y, Han F. Clinical characteristics of recurrent nasopharyngeal carcinoma in high-incidence area. Scientific World Journal. 2012; 2012:719754. https://doi. org/10.1100/2012/719754.

5. Xu T, Tang J, Gu M, Liu L, Wei W, Yang H. Recurrent nasopharyngeal carcinoma: a clinical dilemma and challenge. Curr Oncol. 2013; 20:e406-19.

6. Hua YJ, Han F, Lu LX, Mai HQ, Guo X, Hong MH, Lu TX, Zhao C. Long-term treatment outcome of recurrent nasopharyngeal carcinoma treated with salvage intensity modulated radiotherapy. Eur J Cancer. 2012; 48:3422-28.

7. Kong L, Wang L, Shen C, Hu C, Wang L, Lu JJ. Salvage Intensity-Modulated Radiation Therapy (IMRT) for Locally Recurrent Nasopharyngeal Cancer after Definitive IMRT: A Novel Scenario of the Modern Era. Sci Rep. 2016; 6:32883.

8. Chen C, Fee W, Chen J, Chan C, Khong B, Hara W, Goffinet D, Li D, Le QT. Salvage treatment for locally recurrent nasopharyngeal carcinoma (NPC). Am J Clin Oncol. 2014; 37:327-31.

9. Karam I, Huang SH, McNiven A, Su J, Xu W, Waldron J, Bayley AJ, Kim J, Cho J, Ringash J, Hope A, Chen E, Chan B, et al. Outcomes after reirradiation for recurrent nasopharyngeal carcinoma: north American experience. Head Neck. 2016; 38:E1102-09.

10. Fife BT, Pauken KE, Eagar TN, Obu T, Wu J, Tang Q, Azuma M, Krummel MF, Bluestone JA. Interactions between PD-1 and PD-L1 promote tolerance by blocking the TCR-induced stop signal. Nat Immunol. 2009; 10:1185-92.

11. Carreno BM, Collins M. The B7 family of ligands and its receptors: new pathways for costimulation and inhibition of immune responses. Annu Rev Immunol. 2002; 20:29-53.

12. Kleffel S, Posch C, Barthel SR, Mueller H, Schlapbach C, Guenova E, Elco CP, Lee N, Juneja VR, Zhan Q, Lian CG, Thomi R, Hoetzenecker W, et al. Melanoma Cell-Intrinsic PD-1 Receptor Functions Promote Tumor Growth. Cell. 2015; 162:1242-56.

13. Darb-Esfahani S, Kunze CA, Kulbe H, Sehouli J, Wienert S, Lindner J, Budczies J, Bockmayr M, Dietel M, Denkert C, Braicu I, Jöhrens K. Prognostic impact of programmed cell death-1 (PD-1) and PD-ligand 1 (PD-L1) expression in cancer cells and tumor-infiltrating lymphocytes in ovarian high grade serous carcinoma. Oncotarget. 2016; 7:1486-99. https://doi.org/10.18632/oncotarget.6429.

14. Muenst S, Schaerli AR, Gao F, Däster S, Trella E, Droeser RA, Muraro MG, Zajac P, Zanetti R, Gillanders WE, Weber WP, Soysal SD. Expression of programmed death ligand 1 (PD-L1) is associated with poor prognosis in human breast cancer. Breast Cancer Res Treat. 2014; 146:15-24.

15. Kim JR, Moon YJ, Kwon KS, Bae JS, Wagle S, Kim KM, Park HS, Lee H, Moon WS, Chung MJ, Kang MJ, Jang
KY. Tumor infiltrating PD1-positive lymphocytes and the expression of PD-L1 predict poor prognosis of soft tissue sarcomas. PLoS One. 2013; 8:e82870.

16. Zhou Y, Shi D, Miao J, Wu H, Chen J, Zhou X, Hu D, Zhao C, Deng W, Xie C. PD-L1 predicts poor prognosis for nasopharyngeal carcinoma irrespective of PD-1 and EBVDNA load. Sci Rep. 2017; 7:43627.

17. Zhang J, Fang W, Qin T, Yang Y, Hong S, Liang W, Ma Y, Zhao H, Huang Y, Xue C, Huang P, Hu Z, Zhao Y, Zhang L. Co-expression of PD-1 and PD-L1 predicts poor outcome in nasopharyngeal carcinoma. Med Oncol. 2015; 32:86.

18. Lee VH, Lo AW, Leung CY, Shek WH, Kwong DL, Lam KO, Tong CC, Sze CK, Leung TW. Correlation of PD-L1 Expression of Tumor Cells with Survival Outcomes after Radical Intensity-Modulated Radiation Therapy for NonMetastatic Nasopharyngeal Carcinoma. PLoS One. 2016; 11:e0157969.

19. Hsu MC, Hsiao JR, Chang KC, Wu YH, Su IJ, Jin YT, Chang $\mathrm{Y}$. Increase of programmed death-1-expressing intratumoral CD8 $\mathrm{T}$ cells predicts a poor prognosis for nasopharyngeal carcinoma. Mod Pathol. 2010; 23:1393-403.

20. Flemming A. Cancer: PD1 makes waves in anticancer immunotherapy. Nat Rev Drug Discov. 2012; 11:601.

21. Yang Y, Pang Z, Ding N, Dong W, Ma W, Li Y, Du J, Liu Q. The efficacy and potential predictive factors of PD-1/PD-L1 blockades in epithelial carcinoma patients: a systematic review and meta analysis. Oncotarget. 2016; 7:74350-61. https://doi.org/10.18632/oncotarget.11291.

22. Jain A, Chia WK, Toh HC. Immunotherapy for nasopharyngeal cancer-a review. Linchuang Zhongliuxue Zazhi. 2016; 5:22.

23. Spain L, Larkin J. Combination immune checkpoint blockade with ipilimumab and nivolumab in the management of advanced melanoma. Expert Opin Biol Ther. 2016; 16:389-96.

24. Fehrenbacher L, Spira A, Ballinger M, Kowanetz M, Vansteenkiste J, Mazieres J, Park K, Smith D, Artal-Cortes A, Lewanski C, Braiteh F, Waterkamp D, He P, et al, and POPLAR Study Group. Atezolizumab versus docetaxel for patients with previously treated non-small-cell lung cancer (POPLAR): a multicentre, open-label, phase 2 randomised controlled trial. Lancet. 2016; 387:1837-46.

25. Pitt JM, Vétizou M, Daillère R, Roberti MP, Yamazaki T, Routy B, Lepage P, Boneca IG, Chamaillard M, Kroemer G, Zitvogel L. Resistance Mechanisms to Immune-Checkpoint Blockade in Cancer: Tumor-Intrinsic and -Extrinsic Factors. Immunity. 2016; 44:1255-69.

26. Noman MZ, Desantis G, Janji B, Hasmim M, Karray S, Dessen P, Bronte V, Chouaib S. PD-L1 is a novel direct target of HIF-1 $\alpha$, and its blockade under hypoxia enhanced MDSC-mediated T cell activation. J Exp Med. 2014; 211:781-90. 
27. Locatelli F, Fishbane S, Block GA, Macdougall IC. Targeting Hypoxia-Inducible Factors for the Treatment of Anemia in Chronic Kidney Disease Patients. Am J Nephrol. 2017; 45:187-99.

28. Zhao S, Wu J. Hypoxia inducible factor stabilization as a novel strategy to treat anemia. Curr Med Chem. 2013; 20:2697-711.

29. Lu J, Lee-Gabel L, Nadeau MC, Ferencz TM, Soefje SA. Clinical evaluation of compounds targeting PD-1/PD-L1 pathway for cancer immunotherapy. J Oncol Pharm Pract. 2015; 21:451-67.

30. Zhang Y, Kang S, Shen J, He J, Jiang L, Wang W, Guo Z, Peng G, Chen G, He J, Liang W. Prognostic significance of programmed cell death 1 (PD-1) or PD-1 ligand 1 (PD-L1) Expression in epithelial-originated cancer: a meta-analysis. Medicine (Baltimore). 2015; 94:e515.

31. Pardoll DM. The blockade of immune checkpoints in cancer immunotherapy. Nat Rev Cancer. 2012; 12:252-64.

32. Odorizzi PM, Pauken KE, Paley MA, Sharpe A, Wherry EJ. Genetic absence of PD-1 promotes accumulation of terminally differentiated exhausted CD8+ T cells. J Exp Med. 2015; 212:1125-37.

33. Ghadjar P, Pöttgen C, Joos D, Hayoz S, Baumann M, Bodis S, Budach W, Studer G, Stromberger C, Zimmermann F, Kaul D, Plasswilm L, Olze H, et al. Haemoglobin and creatinine values as prognostic factors for outcome of concurrent radiochemotherapy in locally advanced head and neck cancers : secondary results of two European randomized phase III trials (ARO 95-06, SAKK 10/94). Strahlenther Onkol. 2016; 192:552-60.

34. Liang XX, Li Q, Su Z, Lan XW, Ouyang PY, Mao YP, Shi DB, Deng WG, Cheng ZB, Wang SY, Xie FY. Significant prognostic impact of chemoradiotherapyinduced hemoglobin decrease on treatment outcomes of nasopharyngeal carcinoma. J Cancer. 2015; 6:502-10.

35. Hong B, Lui VW, Hashiguchi M, Hui EP, Chan AT. Targeting tumor hypoxia in nasopharyngeal carcinoma. Head Neck. 2013; 35:133-45.

36. Masoud GN, Li W. HIF-1 $\alpha$ pathway: role, regulation and intervention for cancer therapy. Acta Pharm Sin B. 2015; 5:378-89.
37. Chang YL, Yang CY, Lin MW, Wu CT, Yang PC. High co-expression of PD-L1 and HIF-1 $\alpha$ correlates with tumour necrosis in pulmonary pleomorphic carcinoma. Eur $\mathrm{J}$ Cancer. 2016; 60:125-35.

38. Chen TC, Wu CT, Wang CP, Hsu WL, Yang TL, Lou PJ, Ko JY, Chang YL. Associations among pretreatment tumor necrosis and the expression of HIF-1 $\alpha$ and PD-L1 in advanced oral squamous cell carcinoma and the prognostic impact thereof. Oral Oncol. 2015; 51:1004-10.

39. Barsoum IB, Smallwood CA, Siemens DR, Graham CH. A mechanism of hypoxia-mediated escape from adaptive immunity in cancer cells. Cancer Res. 2014; 74:665-74.

40. Koh J, Jang JY, Keam B, Kim S, Kim MY, Go H, Kim TM, Kim DW, Kim CW, Jeon YK, Chung DH. EML4-ALK enhances programmed cell death-ligand 1 expression in pulmonary adenocarcinoma via hypoxia-inducible factor (HIF)-1 $\alpha$ and STAT3. OncoImmunology. 2015; 5:e1108514.

41. Tian YM, Tian YH, Zeng L, Liu S, Guan Y, Lu TX, Han F. Prognostic model for survival of local recurrent nasopharyngeal carcinoma with intensity-modulated radiotherapy. Br J Cancer. 2014; 110:297-303.

42. Yan F, Ye Z, Wang F, Wang L, Li W, Fu Z. Clinical and imaging characteristics of 53 ulcers of post-radiation nasopharyngeal necrosis in patients with nasopharyngeal carcinoma. Mol Clin Oncol. 2016; 5:351-56.

43. Hua YJ, Chen MY, Qian CN, Hong MH, Zhao C, Guo L, Guo X, Cao KJ. Postradiation nasopharyngeal necrosis in the patients with nasopharyngeal carcinoma. Head Neck. 2009; 31:807-12.

44. Marx RE. Osteoradionecrosis: a new concept of its pathophysiology. J Oral Maxillofac Surg. 1983; 41:283-88.

45. Chen MY, Wang SL, Zhu YL, Shen GP, Qiu F, Luo DH, Chen QY, Jiang R, Cao KJ, Qian CN, Hong MH. Use of a posterior pedicle nasal septum and floor mucoperiosteum flap to resurface the nasopharynx after endoscopic nasopharyngectomy for recurrent nasopharyngeal carcinoma. Head Neck. 2012; 34:1383-88. 